Ingeniería

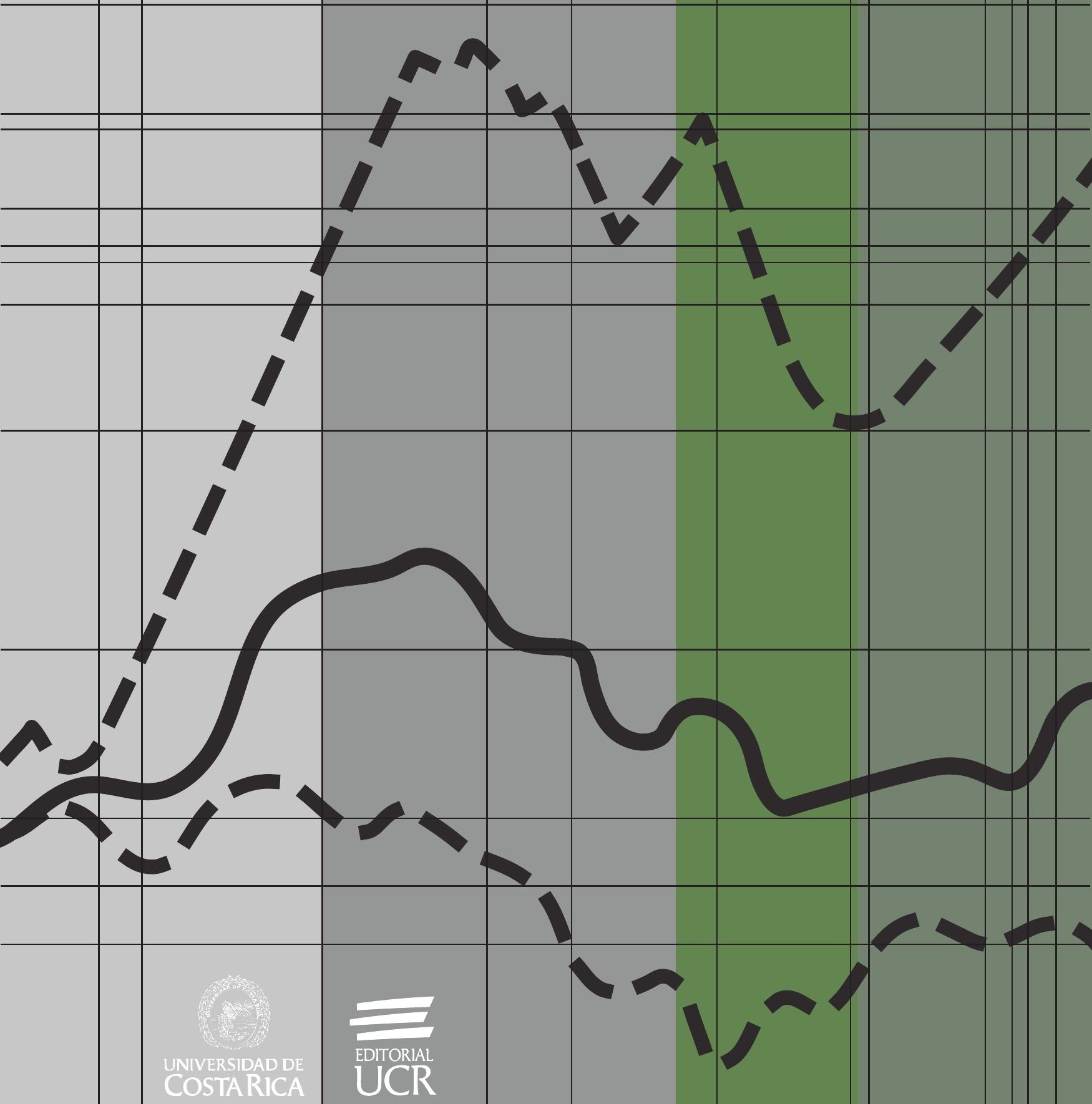




\title{
Clasificación de las rutas de la red de transporte público modalidad autobús de Costa Rica
}

\author{
Classification of the Public Bus Transportation \\ Networks in Costa Rica \\ Cristhian Santiago Quirós-Calderón \\ Universidad de Costa Rica, Escuela de Ingeniería Civil, Programa de Investigación \\ en Desarrollo Urbano Sostenible, (ProDUS-UCR), Costa Rica \\ cristhian.quiros@ucr.ac.cr \\ Jonathan Agüero-Valverde \\ Universidad de Costa Rica, Escuela de Ingeniería Civil, Programa de Investigación \\ en Desarrollo Urbano Sostenible, (ProDUS-UCR), Costa Rica \\ jonathan.aguero@ucr.ac.cr
}

Recibido: 16 de octubre 2018

Aceptado: 23 de abril 2018

\begin{abstract}
Resumen
Este artículo presenta una metodología utilizada para la clasificación de las distintas rutas que componen la totalidad de la red de transporte público modalidad autobús de Costa Rica. El procedimiento se realiza a partir de la necesidad de identificar rutas con problemas tarifarios que terminan por trasladar los costos de operación a los usuarios, para lo cual la desagregación en subgrupos a partir de características comunes facilita la tarea. Debido a la carencia de información relacionada con las características de operación de la red nacional de transporte público, la categorización se realiza aprovechando las variables con la mayor cantidad de información disponible, en este caso: ubicación, estructura, extensión y pendiente. El proceso consiste en la combinación de los subgrupos de las variables seleccionadas, en un orden de jerarquía, con el objetivo de llevar a cabo la agrupación de ramales que presenten comportamientos similares. Como resultado se obtienen grupos más homogéneos, reflejado en la reducción de la dispersión de las distintas variables analizadas. La agrupación permitiría en investigaciones posteriores, la identificación de rutas anómalas en cuanto a parámetros operativos, de especial interés para el análisis de tarifas y el beneficio de los usuarios.
\end{abstract}

Palabras clave:

Transporte Público, Tarifa, Clasificación, Bus, Categorías, Rutas

\begin{abstract}
This paper presents a methodology used for the classification of the public bus transportation networks in Costa Rica. To identify bus routes with fare inconsistency, that affect groups who
\end{abstract}


depend on public transit, smaller groups with similar operational characteristics were created. Due to the lack of information of the public bus transportation network variables, the clustering was made with the most complete characteristics in terms of information availability: location, structure, length and terrain slope. The method consists in the combination of the smaller groups within each variable, with a specific hierarchy, to group the routes with similar characteristics and to simplify the identification of singular routes in terms of fare. As a result, the homogeneity of the smaller groups increases compared to the whole network, as shown in the variables dispersion. The grouping could help identify outliers within the route clusters, being the operational characteristics the ones to address to intervene fare issues.

Key words:

Public Transportation, Fare, Classification, Bus, Categories, Routes 


\section{INTRODUCCIÓN}

Costa Rica presenta una estructura desordenada en su red de transporte público modalidad autobús. Si bien existe muy buena cobertura del territorio nacional por parte del sistema, la regulación, disposición y funcionamiento de la red no es el óptimo, lo que dificulta la continua evaluación y fiscalización de los diversos componentes del engranaje.

Diversos estudios dirigidos a rutas puntuales (Programa de Investigación en Desarrollo Urbano Sostenible, 2014; 2015), han demostrado que existen carencias en la estructura operativa del sistema, cuyos costos se trasladan directamente a la población, perjudicando de esta manera al usuario último de la red. Es necesario contar con una herramienta para identificar diferencias tarifarias, en rutas que operativamente presentan características similares; sobre todo en un sistema como el costarricense, en el cual la totalidad de las rutas es regulada por una misma entidad cuya capacidad de fiscalizar toda la red es limitada.

Debido al elevado costo de realizar auditorías específicas para cada ruta, existe una importante carencia de información respecto a la red de transporte público modalidad autobús del país y se vuelve necesario encontrar metodologías que puedan facilitar la evaluación de las rutas a un nivel más general. Para esto, se debe aprovechar la información existente y disponible de las distintas rutas, como ubicación, estructura física, extensión, tarifa, frecuencia, pasajeros reportados, cantidad de viajes realizados, entre otros que puedan complementar el análisis (Programa de Investigación en Desarrollo Urbano Sostenible, 2016).

La metodología propuesta busca crear categorías a partir de características comunes entre los distintos recorridos, aprovechando de esta manera la mayor cantidad de información disponible. La herramienta permitiría identificar en cada grupo los ramales con comportamiento distinto, para luego realizar análisis independientes a las rutas seleccionadas en miras de corregir las características de operación anómalas.

El uso de agrupamientos en transporte público ya fue explorado por Karlaftis y McCarthy (2002), los cuales, mediante el uso de análisis de grupos desarrollaron una función de costos para una muestra heterogénea de rutas. Mediante la identificación de características similares, dividieron la información en grupos homogéneos pero diferentes entre sí. Hicieron uso de variables como flota, ingreso, cantidad de empleados, distancia recorrida y cantidad de pasajeros, entre otros. Análisis similares se pueden observar en los trabajos de Hartgen y Segedy (1996) y Fielding, Brenner y Faust (1985).

La categorización se realiza con la totalidad de la red nacional, a partir de las variables de ubicación, estructura física, pendiente y extensión, las cuales son las características elegidas al ser las que representan variables importantes desde el punto de vista operativo y cuentan con más información en la base de datos disponible de las rutas de transporte público modalidad autobús.

El proceso de categorización consiste en la combinación de los subgrupos de las variables seleccionadas, en un orden de jerarquía, con el objetivo de llevar a cabo la agrupación de ramales que presenten comportamientos homogéneos, que faciliten la identificación de rutas con comportamientos atípicos respecto a la tarifa cobrada. El análisis a mayor profundidad de los grupos creados permite identificar las desigualdades a nivel de tarifa, de manera que las deficiencias identificadas a nivel operativo se 
puedan tratar por parte de la autoridad reguladora correspondiente y así ajustar cómo se trasladan los costos operativos del servicio a la tarifa cobrada a la población. Ejemplos de evaluaciones similares se pueden observar en los trabajos de Fielding, Babitsky y Brenner (1985) y Chu, Fielding y Lammar (1992).

El propósito de este artículo es proponer una metodología de clasificación de las rutas de transporte público en categorías cuando la disponibilidad de información del esquema operativo sea limitada. El enfoque del proceso de categorización se basa en agrupar las rutas a partir de características físicas comunes, para que en investigaciones posteriores se identifiquen los recorridos con comportamiento distinto al del grupo y se proceda a realizar evaluaciones individuales.

\section{METODOLOGÍAS}

El análisis se realiza mediante el uso de sistemas de información geográfica (SIG), específicamente con el software ArcGIS. Se utilizaron 1235 ramales de la totalidad del país para realizar el proceso de categorización. Cada ruta, al estar representada en SIG, contiene información geográfica asociada, como longitud, ubicación y configuración física, así como otros parámetros que se pueden estimar a partir del programa. Los parámetros operativos de frecuencia, horario, cantidad de pasajeros y tarifa se asociaron a la base de datos de las rutas de SIG y se procesaron por aparte. En la figura 1 se pueden observar las rutas utilizadas para el análisis; el detalle se aprecia a nivel nacional y a nivel del Gran Área Metropolitana.

Como se mencionó anteriormente, la categorización de las rutas se realiza con las variables seleccionadas de manera jerárquica, de manera que se refleje de la mejor manera el comportamiento operativo de cada una de las rutas y su relación con el resto de las rutas del país. El primer paso dentro de esta metodología es la selección de las variables para la categorización.

\subsection{Selección de variables para la categorización}

Basado en la justificación anterior, para cada sistema se deben identificar las variables que sean más representativas para la red, tanto por su importancia como variable como por su disponibilidad. Las variables pueden ser tanto físicas como de operación. Entre las primeras se pueden destacar variables como ubicación, longitud, pendiente y estructura. Entre las variables de operación, se pueden mencionar horario, frecuencia, capacidad, cantidad de unidades, tarifa, cantidad de pasajeros mensuales y ocupación promedio, entre otros. En la presente investigación, tuvo un peso importante la disponibilidad de información, por lo que se eligieron las características físicas de las rutas de transporte público para realizar la clasificación. Las variables utilizadas se describen a continuación:

\section{Ubicación}

La ubicación depende de cada zona de estudio, sin embargo, se deben tener en cuenta características distintivas de las regiones estudiadas. El presente estudio se basó en las siguientes características para la categorización: 
- Jerarquía de ciudades: se establece la ciudad o las ciudades principales de la zona de estudio y en un orden definido de jerarquía, las ciudades primarias, secundarias y terciarias. De ser necesario se establecen otros centros de importancia. Esto permite identificar el tipo de conexión entre ciudades mediante rutas de transporte público y agruparlas a partir de este parámetro.

- Zonas urbanas y rurales: la dinámica de transporte entre zonas urbanas y rurales es muy diferente, sobre todo en cuanto a población movilizada, tipo de unidades y distancia entre paradas.

- Sistemas internos: en ocasiones las ciudades poseen sistemas de transporte público internos, que no poseen relación ni conexión con los otros centros de importancia, los cuales, de igual manera, deben tratarse por separado.

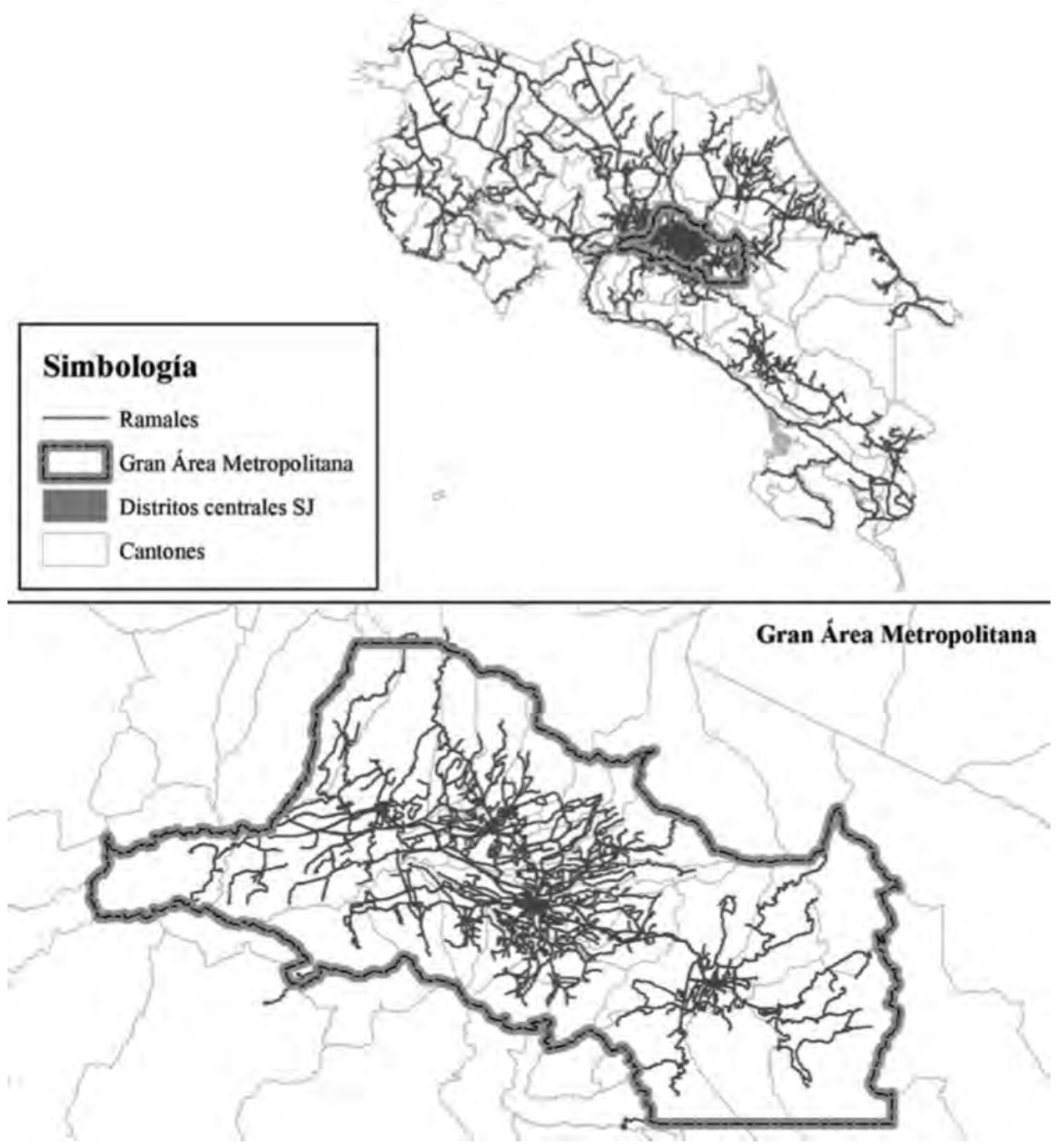

Figura 1. Configuración física de las rutas analizadas. 


\section{Estructura}

Molinero (1997) detalla las estructuras radiales, diametrales, tangenciales, rutas con lazo en un extremo y circulares como los tipos fundamentales de rutas. Costa Rica carece de rutas tangenciales y sus únicas rutas diametrales corresponden a las 6 rutas intersectoriales que operan en la capital, por lo que para la clasificación de los recorridos se utilizó la siguiente descripción:

- Radiales: rutas que viajan de la periferia a los centros poblacionales con flujos direccionales marcados. Se incluye la variación de rutas con un lazo en un extremo, ya que por definición también son rutas radiales.

- Circulares o periféricas: viajan por la periferia de ciudades o poblados importantes.

La figura 2 muestra un ejemplo de una ruta radial corta y una periférica en el centro de San José. Se observa como la ruta radial tiene su origen en uno de los cuatro distritos centrales del cantón, configuración predominante en el sistema de transporte nacional. Con la carencia de rutas troncales, este tipo de estructura presenta alta ineficiencia debido a la acumulación de ramales en corredores comunes, sobre todo en el centro y en las cercanías de este.

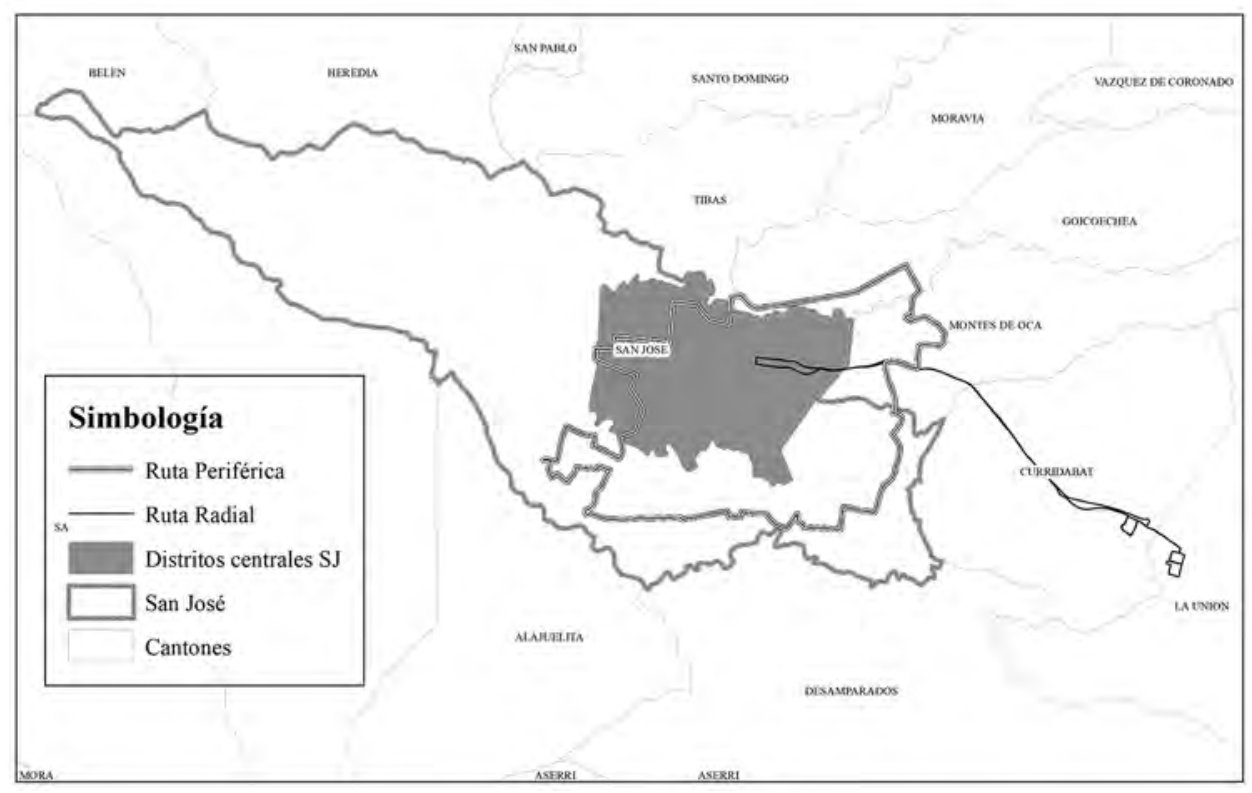

Figura 2. Ejemplo de configuración física de las rutas analizadas.

\section{Pendiente}

La topografía de la zona de estudio influye tanto en los costos como en la velocidad de operación de las distintas rutas. No se pueden agrupar las rutas que operan en llanuras o zonas costeras con las que operan en relieves más quebrados, a pesar de poseer categorías de ubicación y estructura similares. El Manual Centroamericano de Diseño Geométrico (Secretaría de Integración Económica Centroamericana, 2011) 
clasifica la pendiente del terreno como plana, ondulada y montañosa, estableciendo límites de 5\%, $15 \%$ y $30 \%$ de pendiente respectivamente. Sin embargo, un ramal no mantiene una misma categoría de pendiente a lo largo del recorrido; por lo tanto, se establecieron nueve grupos de ramales según la pendiente predominante. Cuando la pendiente predomina en más del 66,6\% del recorrido, este se describe únicamente por un tipo de pendiente, por ejemplo, plana, ondulada o montañosa. Si el ramal no tiene una pendiente que represente más de dos tercios, se describe con los dos tipos de pendiente predominantes en orden de magnitud, por ejemplo, plana-ondulada, plana-montañosa, ondulada-plana, ondulada-montañosa, montañosa-plana, y montañosa-ondulada. Para no crear una excesiva cantidad de subgrupos, se procedió a simplificar la categoría de pendiente de nueve grupos a dos grupos:

- Plana-ondulada: la componen los subgrupos de pendiente plana, plana-ondulada, plana-montañosa y ondulada-plana.

- Ondulada-montañosa: la componen los subgrupos de pendiente ondulada-plana, ondulada, montañosa-plana, montañosa-ondulada y montañosa.

\section{Longitud}

Respecto a la longitud, se debe conocer la distribución de los datos de todos los ramales, de manera que se puedan crear categorías acordes a la composición de las rutas en el país, diferenciando así los servicios urbanos de los interurbanos en distintos rangos. Para homologar criterios, la clasificación tomó en cuenta la metodología empleada en el Consejo de Transporte Público (Ministerio de Obras Públicas y Transporte, 2002), donde el recorrido urbano es menor a los 20 kilómetros en un sentido y el interurbano superior a los 20 kilómetros en un solo sentido.

De las variables categóricas, como el caso de ubicación, estructura y pendiente, se obtuvo la distribución porcentual para comprender el comportamiento de los ramales y así ayudar a la creación de los grupos. Se buscó evitar crear categorías con gran cantidad o con muy poca cantidad de datos. En el caso de las variables cuantitativas, como la longitud, se obtuvieron las estadísticas descriptivas como valor máximo y mínimo, promedio, desviación estándar y los cuartiles para comprender el comportamiento de los datos y posteriormente clasificar la información en grupos que facilitaran la categorización al combinarlo con las demás variables en análisis.

\subsection{Categorización}

Se consideraron los siguientes criterios para el agrupamiento:

- Se establece una jerarquía para las variables seleccionadas.

- Se establecen 30 categorías como el número máximo de grupos.

- Se permite la simplificación de subgrupos de datos de cada variable seleccionada, ya sea por eliminación o unión, de manera que se reduzcan la cantidad de grupos creados.

- Se busca que cada categoría concentre entre el $1 \%$ y el $10 \%$ de la información, de manera que no existan categorías con muy pocos o con muchos datos. 
- Se deben considerar posibles excepciones a la jerarquía, subgrupos y cantidad de datos planteados.

En resumen, la categorización consiste en la combinación de los subgrupos de las variables seleccionadas, en un orden de jerarquía, los cuales se someten a un proceso iterativo en el que se busca reducir la combinación de factores a aproximadamente 30 grupos principales, con el objetivo de que los ramales de cada grupo presenten comportamientos homogéneos, en relación con las demás variables operativas no seleccionadas para la categorización, pero de gran importancia para el esquema operativo de la red de transporte público modalidad autobús.

\subsection{Evaluación de los grupos}

Para la evaluación de la clasificación, se utilizan datos de variables cuantitativas que permitan la comparación entre grupos y dentro de cada grupo mediante la obtención de estadísticas descriptivas como valores mínimos y máximos, promedio, desviación estándar, coeficiente de variación y percentiles.

La dispersión de los datos de cada variable permite determinar qué tan homogéneo es el grupo creado e indica si es necesario realizar ajustes al proceso de categorización, lo cual se ve reflejado mediante valores elevados de desviación estándar y de los coeficientes de variación. Se debe prestar especial atención a los valores extremos, ya que estos son indicadores de rutas con comportamiento atípico dentro de un mismo grupo, que se puede deber tanto a problemas de agrupamiento como a problemas del esquema operativo del ramal en cuestión.

\section{DATOS}

La base de datos con la que se realizó la categorización contiene información de cada uno de los ramales inscritos en el Consejo de Transporte Público (CTP) y regulados por la Autoridad Reguladora de los Servicios Públicos (ARESEP). Del CTP se obtuvo información de horario, frecuencia, capacidad y cantidad de unidades y de ARESEP información de tarifa, demanda mensual y cantidad de carreras mensuales. De los recorridos trabajados en sistemas de información geográfica se extrajo la longitud, la pendiente, la estructura física y la ubicación.

La información más completa corresponde a la obtenida en SIG: 1235 ramales de los 1591 recopilados cuentan con los datos, mientras que, si se incorpora la información del CTP o ARESEP, la cantidad de ramales con los datos completos disminuye drásticamente. Por ende, se trabaja con los 1235 ramales que representan el 77,6\% del total.

\subsection{Consideraciones de las variables utilizadas para la categorización}

\section{Ubicación}

La red de transporte público modalidad autobús de Costa Rica tiene una estructura en su mayoría radial, debido a que los centros de ciudad más importantes son también 
los centros de distribución del transporte público. Estos centros de ciudad funcionan como punto de partida o llegada para los distintos ramales que componen el sistema. La clasificación por ubicación debe ir entonces subordinada al nivel de importancia de los centros de ciudad; sin embargo, el país no cuenta con una jerarquización oficial de ciudades o centros de población, razón por la cual, con apoyo de las investigaciones de Hall (1974), Pujol (2002), y el origen y destino de los ramales analizados, se establece el siguiente orden de jerarquía aplicado a transporte público:

1. Ciudad principal: San José

2. Ciudades primarias: Alajuela, Cartago y Heredia

3. Ciudades secundarias: San Isidro del General, Ciudad Quesada, San Ramón, Turrialba, Liberia, Puntarenas, Limón, Guápiles y Upala

4. Ciudades terciarias: Palmares, Grecia, Naranjo, Los Chiles, Nicoya, Cañas, Esparza, Ciudad Neilly y Golfito

5. Otros centros de distribución: Fortuna, Santa Cruz, Bagaces, Tilarán, Las Juntas, Quepos, Puerto Jiménez, San Vito y Ciudad Cortés

\section{Longitud}

La longitud de los ramales, al ser una variable cuantitativa, requirió de análisis previo antes de crear los grupos para la categorización. La siguiente tabla muestra los estadísticos descriptivos para los ramales registrados. El percentil 75 se ubica en $60 \mathrm{~km}$; sin embargo, el promedio es superior, la desviación estándar es muy elevada y el valor máximo es muy superior a $60 \mathrm{~km}$, lo que indica que se debe explorar la distribución de los datos superiores al percentil 75 para entender su composición.

\section{RESULTADOS}

\subsection{Distribución de variables principales}

Se asignó el nivel uno a San José como el centro de distribución más importante del país, no solo dentro del Gran Área Metropolitana (GAM) (Ministerio de Vivienda y Asentamientos Humanos, 2008), sino en todo el territorio nacional. El nivel dos se asignó para las ciudades que se encuentran dentro del GAM y a su vez son cabecera de provincia. El nivel tres se asignó para las ciudades que se encuentran fuera del GAM y que a su vez son de gran influencia en su zona o región (Salas, 2003), como San Isidro del General en la región Brunca, Ciudad Quesada en la región Huetar Norte y Upala en la Zona Norte - Norte. El nivel cuatro y el nivel cinco se asignaron basados en la cantidad de ramales que dichas ciudades manejan como centro de distribución de transporte público modalidad autobús.

La jerarquización anterior permite clasificar cada ramal según la tabla 2:

De la ubicación se observa que el GAM concentra aproximadamente el $40 \%$ de los ramales del país. Los recorridos con salida desde el centro del San José (AMSJ y SJR) conforman casi la tercera parte de los datos, lo que ilustra la estructura radial a San José de la red de transporte público modalidad autobús del país. Sin embargo, se observa 
cómo también existen gran cantidad de sistemas locales fuera del GAM; los ramales con origen en ciudades de nivel 3 y nivel 4 concentran cerca del $45 \%$ de la totalidad de las rutas, lo cual indica que se deben tratar por aparte. Categorías como AMI, RCP y RDT son ramales que por definición abarcan estructuras que no se pueden incorporar a otros grupos, por ende, incluyen menor cantidad de recorridos.

Tabla 1. Estadísticas descriptivas para la longitud en kilómetros de los ramales

\begin{tabular}{|c|c|}
\hline Mínimo & 2,87 \\
\hline $\mathrm{P}_{25}$ & 14,57 \\
\hline $\mathrm{P}_{50}$ & 26,52 \\
\hline $\mathrm{P}_{75}$ & 59,80 \\
\hline Máximo & 723,36 \\
\hline Promedio & 65,83 \\
\hline Desviación estándar & 112,23 \\
\hline
\end{tabular}

(1) La longitud del recorrido se considera en ambos sentidos

Tabla 2. Clasificación de ramales a partir de los criterios de ubicación

\begin{tabular}{|c|c|c|c|}
\hline Grupo & Código & Nombre & Descripción \\
\hline 1 & AMSJ & Área Metropolitana - San José & $\begin{array}{l}\text { Ramales con origen en ciudades de nivel } 1 \text { y } \\
\text { destinodentro del GAM }\end{array}$ \\
\hline 2 & AMP & Área Metropolitana - Provincia & $\begin{array}{l}\text { Ramales con origen en ciudades de nivel } 2 \text { y destino } \\
\text { dentro del GAM }\end{array}$ \\
\hline 3 & $\mathrm{AMC}$ & Área Metropolitana - Cantón & $\begin{array}{l}\text { Ramales con sistemas locales dentro del GAM que no } \\
\text { tengan origen en ciudades de nivel } 1 \text { ni nivel } 2\end{array}$ \\
\hline 4 & AMI & Área Metropolitana - Interciudad & Ramales que conecten ciudades de nivel 1 y de nivel 2 \\
\hline 5 & SJR & San José - Radial & $\begin{array}{l}\text { Ramales con origen en ciudades de nivel } 1 \text { y destino } \\
\text { fuera del GAM }\end{array}$ \\
\hline 6 & $\mathrm{RCP}$ & Rural Cantón Principal & Ramales que conecten ciudades de nivel 2,3 y 4 \\
\hline 7 & RDP & Rural Distrito Principal & Ramales con origen en ciudades de nivel 3 \\
\hline 8 & RDS & Rural Distrito Secundario & Ramales con origen en ciudades de nivel 4 \\
\hline 9 & RDT & Rural Distrito Terciario & $\begin{array}{l}\text { Ramales con origen en ciudades de nivel } 5 \text { y restantes } \\
\text { ramales no clasificados }\end{array}$ \\
\hline
\end{tabular}


Tabla 3. Distribución de ramales por categoría de ubicación

\begin{tabular}{lll}
\hline Ubicación & Cantidad & Porcentaje \\
\hline AMSJ & 226 & $18,30 \%$ \\
AMP & 182 & $14,74 \%$ \\
AMC & 59 & $4,78 \%$ \\
AMI & 36 & $2,91 \%$ \\
SJR & 117 & $9,47 \%$ \\
RCP & 38 & $3,08 \%$ \\
RDP & 292 & $23,64 \%$ \\
RDS & 267 & $21,62 \%$ \\
RDT & 18 & $1,46 \%$ \\
Total general & 1235 & $100,00 \%$ \\
\hline
\end{tabular}

La distribución de los ramales por categoría de estructura recalca el predominio de la configuración radial en los ramales de la red, razón por la que se debe diferenciar las rutas circulares al tener características distintas.

Tabla 4. Distribución de ramales por categoría de estructura

\begin{tabular}{lcc}
\hline Estructura & Cantidad & Porcentaje \\
\hline Circular & 63 & $5,10 \%$ \\
Radial & 1172 & $94,90 \%$ \\
Total general & 1235 & $100,00 \%$ \\
\hline
\end{tabular}

Se observa el predominio de ramales de pendiente plana con un $41 \%$ de los datos registrados; además, si se consideran todas las rutas con predominio de relieve plano (plana, plana-ondulada, plana-montañosa, ondulada-plana) se observa que alcanzan poco más del $70 \%$ de los recorridos. El porcentaje restante corresponde a rutas en las que predomina el relieve escarpado.

La configuración radial a San José de la red de transporte público modalidad autobús y la escasez de estaciones de trasbordo de alta capacidad en la periferia del GAM y fuera de esta inciden en que existan rutas de tan elevada longitud. Rutas que varíen de $60 \mathrm{~km}$ hasta $723 \mathrm{~km}$ presentan amplio rango de variación en su esquema operativo, el cual puede 
ser completamente diferente en aspectos de estructura tarifaria, polígonos de carga de pasajeros y poblaciones atendidas. Por ende, como se muestra en la tabla 6 , se crean cuatro categorías superiores al percentil 75 para entender el comportamiento de dichas rutas.

Tabla 5. Distribución de ramales por categoría de pendiente

\begin{tabular}{lcccc}
\hline Pendiente resumida & Pendiente & Cantidad & Porcentaje & Porcentaje resumido \\
& Plana & 509 & $41,21 \%$ & \\
Plana - ondulada & Plana-ondulada & 236 & $19,11 \%$ & $73,68 \%$ \\
& Plana-montañosa & 19 & $1,54 \%$ & \\
& Ondulada-plana & 146 & $11,82 \%$ & \\
& Ondulada & 160 & $12,96 \%$ & $26,32 \%$ \\
Ondulada - montañosa & Montañosa-plana & 34 & $2,75 \%$ & \\
& Ondulada-montañosa & 58 & $4,70 \%$ & \\
& Montañosa-ondulada & 49 & $3,97 \%$ & $100,00 \%$ \\
\hline Total general & Montañosa & 24 & $1,94 \%$ & \\
\hline
\end{tabular}

Tabla 6. Distribución preliminar de ramales por longitud del recorrido.

\begin{tabular}{ccc}
\hline Longitud $(\mathrm{km})^{1}$ & Cantidad & Porcentaje \\
\hline $0-10$ & 144 & $11,66 \%$ \\
$10-20$ & 308 & $24,94 \%$ \\
$20-40$ & 335 & $27,13 \%$ \\
$40-60$ & 140 & $11,34 \%$ \\
$60-100$ & 139 & $11,26 \%$ \\
$100-200$ & 81 & $6,56 \%$ \\
$200-400$ & 40 & $3,24 \%$ \\
\hline Mayor a 400 & 48 & $3,89 \%$ \\
Total general & 1235 & $100,00 \%$ \\
\hline
\end{tabular}

(1)La longitud del recorrido se considera en ambos sentidos

Se observa que poco más del 10\% de los ramales se encuentran entre los $60 \mathrm{~km}$ y los $100 \mathrm{~km}$ de longitud y que de igual manera poco más del 10\% de los ramales 
tienen longitud superior a los $100 \mathrm{~km}$. Sustento con el cual y complementado con los criterios del CTP, se establecieron cuatro grupos de ramales basados en la distribución de longitud, con lo que se obtiene la siguiente división:

Tabla 7. Distribución de grupos definitivos de ramales por longitud del recorrido

\begin{tabular}{cccc}
\hline Descripción & Longitud $(\mathrm{km})^{1}$ & Cantidad & Porcentaje \\
\hline Corto & $0-20$ & 452 & $36,60 \%$ \\
Mediano & $20-40$ & 335 & $27,13 \%$ \\
Largo & $40-125$ & 307 & $24,86 \%$ \\
Muy Largo & Mayor a 125 & 141 & $11,42 \%$ \\
Total general & & 1235 & $100,00 \%$ \\
\hline
\end{tabular}

(1)La longitud del recorrido se considera en ambos sentidos

Como se mencionó anteriormente, los criterios del Ministerio de Obras Públicas y Transportes (MOPT), establecen las rutas de $20 \mathrm{~km}$ de longitud en un solo sentido como urbanas, lo que corresponde a $40 \mathrm{~km}$ de longitud en ambos sentidos según la información de la presente investigación. Las rutas superiores a $40 \mathrm{~km}$ en ambos sentidos se clasifican como interurbanas. La clasificación elegida respeta el límite de $40 \mathrm{~km}$ y a su vez establece un límite superior de $20 \mathrm{~km}$ para las rutas cortas y uno inferior de $125 \mathrm{~km}$ para las rutas muy largas, que permite una mejor distribución de la cantidad de datos.

\subsection{Categorización}

La categorización funciona como un árbol de decisión, en el cual el primer nivel lo compone la variable de ubicación, seguido de las variables de estructura física, pendiente y longitud, respectivamente. Según lo establecido en las tablas 3 a 7, la ubicación se compone de nueve grupos, la estructura física de dos, la pendiente de dos y la longitud de cuatro. Si cada grupo se desagregara al máximo, se obtendría un total de 144 categorías, una cantidad inmanejable para efectos de análisis, la cual no respeta el tope de 30 categorías elegido en los criterios preliminares.

La categorización se realiza basada en un procedimiento ordenado de desagregación de las variables principales y se describe a continuación:

1. Se desagregan los nueve grupos de ubicación en sus 16 subgrupos posibles. La figura 3 muestra los subgrupos completos de la rama radial, plana-ondulada. El último nivel se mantiene igual para la rama ondulada-montañosa y la rama periférica es idéntica a la radial en el penúltimo y último nivel. 


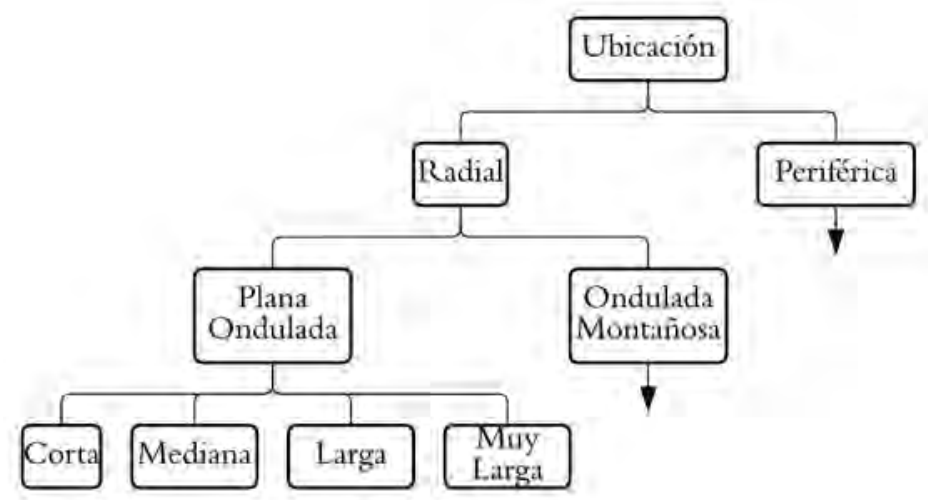

Figura 3. Diagrama de subgrupos posibles para cada grupo de ubicación

2. Por definición, ubicación y características operativas, no todos los grupos de ubicación se desagregan en 16 subgrupos. Por ejemplo, los ramales RSJ son radiales; por ende, solo cuentan con la parte izquierda del diagrama de la figura 3 al excluir la estructura periférica; además, por características operativas su longitud es larga o muy larga, por lo que al final los ramales RSJ se subdividen en únicamente 4 grupos posibles:

a. Radial, plana - ondulada, larga.

b. Radial, plana - ondulada, muy larga.

c. Radial, ondulada - montañosa, larga.

d. Radial, ondulada - montañosa, muy larga.

3. Después de esta desagregación se obtienen 56 de 144 grupos posibles.

4. Posteriormente, se analizan los grupos resultantes al último nivel y los que posean porcentajes menores al $1 \%$ de la totalidad de los datos, se descartan como categorías. Ante esto se tienen dos opciones, que a su vez se ilustran en la tabla 11 para el caso de los ramales AMP y los ramales AMC:

a. Se unen subgrupos del mismo estrato de longitud similar hasta alcanzar el $1 \%$.

b. Se elimina el último nivel de clasificación.

De la tabla 8 se observa cómo en ambas categorías de pendiente, los ramales de longitud larga cuentan con menos del $1 \%$ del total de los datos, por lo que se añaden a los de longitud mediana para formar un nuevo subgrupo que sí supera el porcentaje mínimo de $1 \%$. Después del análisis la cantidad de grupos se reduce a 35 de 144 posibles. 
Tabla 8. Subgrupos para los ramales con categoría de ubicación AMP

\begin{tabular}{|c|c|c|c|c|c|c|}
\hline Ubicación & \multicolumn{6}{|c|}{ AMP } \\
\hline Estructura & \multicolumn{6}{|c|}{ Radial } \\
\hline Pendiente & \multicolumn{3}{|c|}{ Ondulada - Montañosa } & \multicolumn{3}{|c|}{ Plana - Ondulada } \\
\hline Longitud & \multicolumn{3}{|c|}{ ana } & \multicolumn{2}{|r|}{ ana } & Larga \\
\hline Ramales & 23 & 17 & 3 & 78 & 37 & 12 \\
\hline Porcentaje del total & $1.86 \%$ & $1.38 \%$ & $0.24 \%$ & $6.32 \%$ & $3.00 \%$ & $0.97 \%$ \\
\hline Porcentaje del grupo & $1.86 \%$ & & $2 \%$ & $7.29 \%$ & & \\
\hline
\end{tabular}

Tabla 9. Subgrupos para los ramales con categoría de ubicación AMC

\begin{tabular}{ccccc}
\hline Ubicación & \multicolumn{4}{c}{ AMC } \\
Estructura & \multicolumn{3}{c}{ Radial } \\
Pendiente & Ondulada - Montañosa & Plana - Ondulada \\
Longitud & Corta & Mediana & Corta & Mediana \\
Ramales & 20 & 4 & 16 & 2 \\
Porcentaje del total & $1.62 \%$ & $0.32 \%$ & $1.30 \%$ & $0.16 \%$ \\
Porcentaje del grupo & & $1.94 \%$ & & $1.46 \%$ \\
\hline
\end{tabular}

De la tabla 9 se observa cómo, al igual que en el caso anterior, en ambas categorías de pendiente los ramales de longitud mediana cuentan con menos del $1 \%$ del total de los datos. Sin embargo, al solo existir dos subgrupos de longitud (corta y mediana), se decide no tomar en cuenta el último nivel de clasificación (longitud) para establecer las categorías.

5. Se procede de la misma manera para el penúltimo nivel (pendiente), tal como se describió en el punto cuatro para el último nivel (longitud). Se analizan los grupos resultantes al penúltimo nivel, y los que posean porcentajes menores al $1 \%$ de la totalidad de los datos se descartan como categorías. Al tener únicamente dos grupos de pendiente (plana - ondulada y ondulada - montañosa), si se cumple la condición anterior, se elimina el penúltimo nivel de clasificación para ese grupo de ubicación.

6. Después del análisis la cantidad de grupos se reduce a 31 de 144 posibles. 
Tabla 10. Resumen de categorías creadas y las variables que las construyen.

\begin{tabular}{|c|c|c|c|c|c|c|c|}
\hline Grupo & Código & Localización & Estructura & Pendiente & Longitud & Cantidad & Porcentaje \\
\hline 1 & SJ-P & AMSJ & Periférica & N/A & N/A & 8 & $0,65 \%$ \\
\hline 2 & SJ-RC & AMSJ & Radial & N/A & Corta & 113 & $9,15 \%$ \\
\hline 3 & SJ-RM & AMSJ & Radial & N/A & Mediana-Larga & 105 & $8,50 \%$ \\
\hline 4 & P-P & AMP - AMC & Periférica & N/A & $\mathrm{N} / \mathrm{A}$ & 29 & $2,35 \%$ \\
\hline 5 & P-ROC & AMP & Radial & Ondulada - Montañosa & Corta & 23 & $1,86 \%$ \\
\hline 6 & P-ROM & AMP & Radial & Ondulada - Montañosa & Mediana & 20 & $1,62 \%$ \\
\hline 7 & P-RPC & AMP & Radial & Plana - Ondulada & Corta & 78 & $6,32 \%$ \\
\hline 8 & P-RPM & AMP & Radial & Plana - Ondulada & Mediana & 49 & $3,97 \%$ \\
\hline 9 & $\mathrm{C}-\mathrm{RP}$ & $\mathrm{AMC}$ & Radial & Plana - Ondulada & N/A & 24 & $1,94 \%$ \\
\hline 10 & C-RO & $\mathrm{AMC}$ & Radial & Ondulada - Montañosa & $\mathrm{N} / \mathrm{A}$ & 18 & $1,46 \%$ \\
\hline 11 & AMI & AMI & Radial & $\mathrm{N} / \mathrm{A}$ & $\mathrm{N} / \mathrm{A}$ & 36 & $2,91 \%$ \\
\hline 12 & R-ROL & RSJ & Radial & Ondulada - Montañosa & Larga & 22 & $1,78 \%$ \\
\hline 13 & R-ROML & RSJ & Radial & Ondulada - Montañosa & Muy Larga & 23 & $1,86 \%$ \\
\hline 14 & $\mathrm{R}-\mathrm{RP}$ & RSJ & Radial & Plana - Ondulada & $\mathrm{N} / \mathrm{A}$ & 72 & $5,83 \%$ \\
\hline 15 & $\mathrm{RCP}$ & $\mathrm{RCP}$ & Radial & $\mathrm{N} / \mathrm{A}$ & N/A & 38 & $3,08 \%$ \\
\hline 16 & DP-P & RDP & Periférica & N/A & $\mathrm{N} / \mathrm{A}$ & 20 & $1,62 \%$ \\
\hline 17 & DP-ROC & RDP & Radial & Ondulada - Montañosa & Corta & 26 & $2,11 \%$ \\
\hline 18 & DP-ROL & RDP & Radial & Ondulada - Montañosa & Larga & 24 & $1,94 \%$ \\
\hline 19 & DP-ROM & RDP & Radial & Ondulada - Montañosa & Mediana & 24 & $1,94 \%$ \\
\hline 20 & DP-RPC & RDP & Radial & Plana - Ondulada & Corta & 42 & $3,40 \%$ \\
\hline 21 & DP-RPL & RDP & Radial & Plana - Ondulada & Larga & 82 & $6,64 \%$ \\
\hline 22 & DP-RPM & RDP & Radial & Plana - Ondulada & Mediana & 54 & $4,37 \%$ \\
\hline 23 & DP-RPML & RDP & Radial & Plana - Ondulada & Muy Larga & 20 & $1,62 \%$ \\
\hline 24 & DS-ROC & RDS & Radial & Ondulada - Montañosa & Corta & 46 & $3,72 \%$ \\
\hline 25 & DS-ROM & RDS & Radial & Ondulada - Montañosa & Mediana & 28 & $2,27 \%$ \\
\hline 26 & DS-ROL & RDS & Radial & Ondulada - Montañosa & Larga & 44 & $3,56 \%$ \\
\hline 27 & DS-RPC & RDS & Radial & Plana - Ondulada & Corta & 32 & $2,59 \%$ \\
\hline 28 & DS-RPL & RDS & Radial & Plana - Ondulada & Larga & 78 & $6,32 \%$ \\
\hline 29 & DS-RPM & RDS & Radial & Plana - Ondulada & Mediana & 39 & $3,16 \%$ \\
\hline 30 & RDT & RDT & Radial & N/A & N/A & 18 & $1,46 \%$ \\
\hline
\end{tabular}


7. Como se mencionó anteriormente, es muy importante diferenciar los ramales de estructura radial de los ramales de estructura periférica. Sin embargo, cómo se exploró en la desagregación, los porcentajes correspondientes a estructuras periféricas siempre se encuentran por debajo del 1\%, y estos grupos según los criterios establecidos no pueden considerarse como categorías. Por ende, para poder reducir la cantidad de 31 rutas a 30 o menos, en el caso de las estructuras periféricas se aplican dos excepciones que se detallan más adelante.

8. Posterior al análisis, la cantidad de grupos se reduce a 30 de 144 posibles.

La tabla 10 resume las variables utilizadas para la creación de cada categoría y a su vez es la lista de las categorías definitivas. La columna grupo y código corresponden a identificadores cortos para hacer referencia a los grupos en caso de análisis. Las columnas de localización, estructura, pendiente y longitud corresponden a las variables utilizadas para la categorización. Se puede observar que, en caso de no utilizarse una variable para la clasificación, por los motivos mencionados anteriormente, se anota que dicha celda no aplica (N/A).

Del desglose de las categorías se observan tres grupos de ubicación que no requirieron desagregarse: AMI, RCP y RDT. Las rutas interciudades del Área Metropolitana (AMI) por definición son radiales de longitud mediana, y al encontrarse dentro del GAM su pendiente es plana; las rutas rurales de cantón principal (RCP) por definición son radiales de longitud larga o muy larga, por lo que aunque tengan secciones importantes de pendiente ondulada o montañosa, la predominante es la plana; por último, las rutas rurales de distrito terciario (RDT), por su diversidad de composición, al desagregarse, ningún grupo superaba el $1 \%$ requerido.

\section{Excepciones}

En cuanto a la estructura de los ramales, se observa que únicamente tres grupos de los 30 presentan tipo periférica o circular. Los grupos uno y cuatro son los únicos que presentan excepciones en la conformación de la categoría. El grupo uno no supera el 1\% de los datos; sin embargo, al ser su composición tan diferente a la de las rutas radiales del AMSJ y al ser rutas que movilizan una cantidad importante de pasajeros, se decide definirlo como categoría. El grupo cuatro resulta de la unión de las rutas periféricas de los grupos de ubicación AMP y AMC, las cuales, en el caso del grupo AMP, no alcanzaban más del $1 \%$ de datos. Por esto, al ubicarse ambos grupos dentro del GAM se decide unirlas por categoría de estructura antes que de ubicación.

Se considera la presente investigación como un avance en comparación con el único documento que ha abordado la agrupación de rutas por categorías, titulado "Tipos de transporte público colectivo según clasificación de servicio" del Ministerio de Obras Públicas y Transportes de Costa Rica y emitido en el 2002. Dicho documento únicamente clasifica las rutas en seis categorías: Interurbana larga, Urbana Plana, Urbana no Plana, Interurbana Media Plana, Interurbana Corta Plana y Urbana No Plana, a partir de la longitud, tiempo de viaje, velocidad, trazado vertical y trazado horizontal. La consideración de la ubicación en el análisis permite reducir los sesgos de agrupar rutas 
del GAM con rutas fuera del GAM, área que maneja mayores niveles de congestión y distintos escenarios de operación, con mayores frecuencias y pasajeros movilizados.

\subsection{Evaluación de grupos}

El uso de variables secundarias para la evaluación de las categorías permite observar si la clasificación elegida refleja comportamientos dentro de cada grupo distintos a los de otros grupos y así justificar las categorías. De igual manera, permite observar la variación dentro de cada grupo y así evaluar las categorías individualmente.

Se utilizó la tarifa por kilómetro para el análisis:

- Tarifa por kilómetro: monto cobrado por viaje en colones por kilómetro de recorrido del ramal.

Se debe prestar especial atención a la cantidad de datos utilizados para la evaluación. En la tabla 11, un total de 1128 de 1235 ramales contaban con la información de tarifa por kilómetro para la evaluación. Si bien lo ideal es contar con la información completa, es posible obtener resultados satisfactorios del análisis con la información disponible.

En comparación con la totalidad de los datos, se observa cómo casi todas las categorías creadas poseen un coeficiente de variación inferior al global (0.5), lo que implica que dichas agrupaciones presentan comportamientos más homogéneos entre las rutas que las componen. Casos como SJ-RM, R-ROML y AMI presentan valores inferiores a 0.2 , siendo las categorías más homogéneas, cosa que justifica su agrupación. Solamente en tres casos se supera el coeficiente de variación global (0.5): con los grupos DP-ROC, DP-RPL y DS-RPM. Se observa que corresponden a rutas que tienen su origen y destino por fuera del GAM, lo cual puede influir en la actualización tarifaria de estas y por ende en mayores diferencias entre sí. Análisis posteriores deben procurar la disminución del coeficiente de variación por grupo, garantizando mayor homogeneidad en el establecimiento de tarifas.

El percentil 50 y el promedio permiten observar la variación entre grupos. Las categorías con los valores más elevados en la tabla 11 corresponden a rutas de longitud corta, como en el caso de las categorías SJ-RC, P-ROC, C-RP y C-RO en el GAM y DP-ROC, DP-RPC, DS-ROC y DS-RPC fuera del GAM. El caso opuesto ocurre para las rutas de mayor longitud, como en el caso de los ramales radiales a San José R-ROML y R-RP y la categoría DP-RPML de longitud muy larga. Entre los ramales de longitud corta y los de longitud larga se observa hasta el doble de diferencia en la tarifa por kilómetro.

La pendiente juega un papel importante en la tarifa en los grupos de ubicación AMP (2) y RDP (7). Si se comparan los ramales P-ROC y P-ROM de pendiente ondulada montañosa con los ramales P-RPC y P-RPM de pendiente plana - ondulada, se observa que los primeros presentan mayor valor de tarifa por kilómetro, a pesar de P-ROC y P-RPC son ramales de longitud corta y P-ROM y P-RPM de longitud mediana. Mismo caso ocurre al comparar los ramales DP-ROC, DP-ROL y DP-ROM de pendiente ondulada montañosa y de longitud corta, mediana y larga, respectivamente, con los ramales DP-RPC, DP-RPL y DP-RPM de pendiente plana - ondulada y de longitud corta, mediana y larga. 
Tabla 11. Estadísticas descriptivas de la tarifa por kilómetro para cada categoría.

\begin{tabular}{|c|c|c|c|c|c|c|c|c|c|c|c|}
\hline $\begin{array}{l}.00 \\
.00 \\
.0 \\
0 \\
0\end{array}$ & $\stackrel{\stackrel{\Xi}{\Xi}}{\stackrel{\Xi}{\Sigma}}$ & $\curvearrowleft$ & 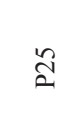 & 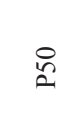 & $\frac{n}{2}$ & 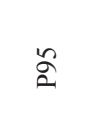 & 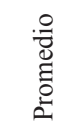 & $\begin{array}{l}\stackrel{\Xi}{\Xi} \\
\text { 离 }\end{array}$ & 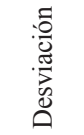 & $\vec{u}$ & 点 \\
\hline SJ-P & 14,36 & 14,36 & 15,02 & 20,04 & 38,6 & . & 24,74 & 41 & 11,65 & 0,47 & 6 \\
\hline SJ-RC & 12,44 & 14,05 & 16,74 & 19,51 & 22,73 & 28,64 & 20,34 & 49,12 & 5,64 & 0,28 & 112 \\
\hline SJ-RM & 7,22 & 10,31 & 11,85 & 13,7 & 15,59 & 17,54 & 13,77 & 21,81 & 2,49 & 0,18 & 101 \\
\hline P-P & 7,74 & 8,29 & 15,23 & 19,56 & 31 & 50,52 & 22,6 & 55,85 & 11,18 & 0,49 & 26 \\
\hline P-ROC & 17,93 & 17,93 & 21,85 & 24,24 & 27,54 & . & 25,29 & 39,78 & 5,71 & 0,23 & 19 \\
\hline P-ROM & 11,17 & 11,17 & 15,68 & 17,71 & 22,37 & . & 19,64 & 39,72 & 6,42 & 0,33 & 19 \\
\hline P-RPC & 13,52 & 14,27 & 16,86 & 21,05 & 26,5 & 38,88 & 23,18 & 74,18 & 9,13 & 0,39 & 71 \\
\hline P-RPM & 6,6 & 7,51 & 10,79 & 13,02 & 15,52 & 21,15 & 13,46 & 22,8 & 3,81 & 0,28 & 46 \\
\hline C-RP & 13,99 & 14,05 & 18,7 & 26,78 & 36,26 & 62,42 & 28,44 & 64,04 & 12,68 & 0,45 & 22 \\
\hline C-RO & 12,53 & 12,53 & 17,21 & 23,98 & 26,87 & . & 23,47 & 42,5 & 7,93 & 0,34 & 16 \\
\hline AMI & 9,4 & 9,68 & 11,79 & 12,44 & 13,05 & 19 & 12,69 & 20,11 & 2,17 & 0,17 & 34 \\
\hline R-ROL & 10,34 & 10,49 & 12,84 & 19,92 & 27,99 & 41,14 & 21,55 & 41,31 & 9,25 & 0,43 & 22 \\
\hline R-ROML & 8,01 & 8,05 & 10,73 & 11,31 & 12,55 & 13,77 & 11,25 & 13,88 & 1,60 & 0,14 & 23 \\
\hline R-RP & 2,67 & 7,05 & 9,01 & 10,14 & 11,39 & 15,47 & 10,54 & 30,51 & 3,29 & 0,31 & 69 \\
\hline $\mathrm{RCP}$ & 3,97 & 6,33 & 9,41 & 11,27 & 14,5 & 16,64 & 11,51 & 17,15 & 3,10 & 0,27 & 36 \\
\hline DP-P & 18,74 & 18,74 & 21,49 & 25,6 & 40,67 & . & 31,84 & 69,74 & 13,80 & 0,43 & 15 \\
\hline DP-ROC & 13,31 & 14,06 & 22,88 & 30,96 & 44,48 & 114,83 & 38,13 & 124,34 & 24,62 & 0,65 & 24 \\
\hline DP-ROL & 8,26 & 8,93 & 15,86 & 20,36 & 27,76 & 33,12 & 20,78 & 33,83 & 6,88 & 0,33 & 23 \\
\hline DP-ROM & 9,24 & 9,34 & 12,83 & 19,63 & 24,29 & 33,63 & 18,96 & 35,02 & 7,07 & 0,37 & 24 \\
\hline DP-RPC & 14,53 & 14,9 & 19,89 & 24,54 & 31,16 & 59,06 & 28,54 & 76,25 & 13,25 & 0,46 & 39 \\
\hline DP-RPL & 7,04 & 10,38 & 13,23 & 16,01 & 19,7 & 46,3 & 19,92 & 59,13 & 11,49 & 0,58 & 53 \\
\hline DP-RPM & 4,88 & 8,46 & 11,26 & 13,59 & 19,21 & 22,23 & 15,20 & 49,15 & 6,07 & 0,40 & 71 \\
\hline DP-RPML & 4,91 & 4,91 & 7,38 & 10,09 & 11,77 & . & 10,36 & 17,21 & 3,27 & 0,32 & 18 \\
\hline DS-ROC & 8,77 & 12,65 & 20,58 & 25,93 & 34,24 & 48,66 & 27,89 & 50,31 & 10,38 & 0,37 & 38 \\
\hline DS-ROM & 3,41 & 4,71 & 13,94 & 17,73 & 21,1 & 23,6 & 16,82 & 27,44 & 5,37 & 0,32 & 42 \\
\hline DS-ROL & 2,43 & 3,66 & 9,15 & 14,67 & 22,01 & 28,61 & 14,99 & 29 & 6,90 & 0,46 & 27 \\
\hline DS-RPC & 12,44 & 12,46 & 18,48 & 24,33 & 36,79 & 62,92 & 28,19 & 68,29 & 13,50 & 0,48 & 28 \\
\hline DS-RPL & 6,15 & 7,06 & 12,88 & 17,65 & 19,98 & 41,91 & 18,92 & 48,68 & 9,40 & 0,50 & 30 \\
\hline DS-RPM & 1,22 & 3,57 & 9,73 & 12,99 & 16,76 & 28,95 & 14,29 & 47,55 & 8,22 & 0,58 & 64 \\
\hline RDT & 9,65 & 9,65 & 13,98 & 16,82 & 20,96 & . & 16,97 & 22,05 & 4,45 & 0,26 & 10 \\
\hline Total & 1,22 & 9,37 & 13,54 & 16,95 & 23,05 & 40,28 & 19,83 & 124,34 & 9,97 & 0,50 & 1128 \\
\hline
\end{tabular}


Se observa cómo en general, la dispersión es menor en los grupos en comparación con el total de los datos. Solo en los grupos DP-ROC, DP-RPL y DS-RPM las categorías presentan un coeficiente de variación superior al general. Esto a su vez coincide con valores extremos presentes en dichas categorías, como el mínimo general de 1,22 colones por kilómetro en la categoría DS-RPM y el máximo general de 124,34 colones por kilómetro en la categoría DP-ROC, lo que indica que, muy probablemente, el identificar y evaluar los valores extremos permitiría disminuir a futuro la desviación dentro de cada grupo.

La creación de categorías es un proceso dinámico y dependiente de la cantidad de información disponible. La continua actualización de la red de transporte público modalidad autobús del país implica la continua actualización de las categorías, de manera que esta sirva como herramienta rápida para la identificación de rutas que presenten anomalías a nivel operativo en comparación con rutas de composición similar.

El procedimiento anterior es de utilidad para regiones con pocos o nulos registros acerca de las características operativas de su red. El utilizar únicamente información física obtenida de SIG, permite elaborar la clasificación teniendo como insumo el recorrido de los ramales y la topografía del terreno a escalas no muy detalladas. Si no se cuenta con la ocupación promedio, que depende de la cantidad de pasajeros y la capacidad del servicio, las cuales son variables que suelen ser más escazas en cuanto a información, se puede utilizar únicamente la tarifa por kilómetro para la evaluación de los grupos e identificación de los valores extremos. La evaluación y corrección de la estructura operativa de los ramales que se identifican como valores extremos permiten el mejoramiento progresivo de la red, en miras de mejorar la equidad y la eficiencia en el transporte público.

El análisis presentado ha sido de utilidad para los entes reguladores de transporte; sus resultados se han utilizado para priorizar la elección de rutas a las cuales elaborarles auditorías específicas y así poder reducir las diferencias operativas que existan dentro de una misma categoría. De igual manera, se pueden aprovechar los insumos generados en SIG junto con indicadores demográficos y económicos para realizar otro tipo de análisis, como lo es el acceso a transporte público por parte de la población.

\section{CONCLUSIONES Y RECOMENDACIONES}

La categorización de la red de transporte público modalidad autobús de Costa Rica define 30 grupos a partir de las siguientes variables en orden de jerarquía: ubicación, estructura física, pendiente y longitud. Cada variable se desagrega en subgrupos que permiten mayor especificidad al crear las distintas categorías. La obtención de 30 grupos a partir de más de 1200 ramales permite un mejor manejo de la información, facilitando el análisis específico por tipo de ruta o por ruta con características anómalas.

Se considera la variable de ubicación como la principal, al existir diferencias muy marcadas principalmente entre los ramales que tienen su origen y destino dentro del GAM y aquellos que tienen ya sea su origen, su destino o ambos fuera del GAM. En el siguiente nivel de jerarquía, la estructura física permite separar de las rutas radiales, las cuales consisten el $95 \%$ de las rutas del país, las rutas periféricas o circulares, cuyo 
funcionamiento es muy distinto al radial. Las últimas categorías de pendiente y longitud permiten realizar la última segregación de los grupos, considerando variables que afectan más directamente el consumo de combustible y materiales de las unidades, orientado al establecimiento de tarifas.

Los grupos creados son dinámicos y se pueden ver sujetos a cambios cuando se actualice la base de datos utilizada para la categorización. Por lo tanto, se recomienda robustecer la base de datos, de manera que todas las rutas del país queden asignadas a una categoría y así favorecer la evaluación del sistema.

La evaluación de la metodología se llevó a cabo con la variable tarifa por kilómetro, debido a la importancia de esta variable para los usuarios del transporte público. Esto permitió corroborar las diferencias entre los promedios de cada grupo, así como la disminución de la dispersión al pasar del análisis general al análisis de cada categoría, validando de esta manera la metodología.

La metodología desarrollada puede ser implementada en otros sistemas de transporte que no cuenten con categorías de ruta o en los que se desee actualizarlos, donde se cuente como mínimo con las rutas digitalizadas para su uso en sistemas de información geográfica. Como se ha mencionado anteriormente, mayor disponibilidad de información podría mejorar la desagregación en otros casos de análisis, sustituyendo incluso algunas de las variables que se utilizaron en el presente estudio. Datos de frecuencia, demanda de pasajeros, velocidad promedio o tiempos de viaje son variables que pueden contribuir con la mejora del procedimiento.

Se recomienda identificar los ramales con valores de tarifa por kilómetro que difieran del comportamiento normal de cada grupo. Esta preselección permite destinar recursos para evaluar las anomalías operativas y proponer soluciones que eviten que dicha problemática se traslade como costos a los usuarios del servicio, ya que, si bien el procedimiento no permite definir tarifas, puede utilizarse como herramienta previa para identificar las rutas prioritarias para el análisis.

\section{REFERENCIAS}

Chu, X., Fielding, G. J., \& Lamar, B. W. (1992). Measuring transit performance using data envelopment analysis. Transportation Research Part A: Policy and Practice, 26(3), 223-230.

Fielding, G. J., Brenner, M. E., \& Faust, K. (1985). Typology for bus transit. Transportation Research Part A: General, 19(3), 269-278.

Fielding, G. J., Babitsky, T. T., \& Brenner, M. E. (1985). Performance evaluation for bus transit. Transportation Research Part A: General, 19(1), 73-82.

Hall, Carolyn (1974), La jerarquía urbana de Costa Rica: una consideración de la aplicación de modelos geográficos. Heredia: Universidad Nacional.

Hartgen, D. T., \& Segedy, J. (1996). Peer groups for transit system performance. Charlotte: University of North Carolina at Charlotte, Center for Interdisciplinary Transportation Studies. 
Karlaftis, M. G., \& McCarthy, P. (2002). Cost structures of public transit systems: a panel data analysis. Transportation Research Part E: Logistics and Transportation Review, 38(1), 1-18.

Ministerio de Vivienda y Asentamientos Humanos (MIVAH). (2008). Plan Regional Urbano de la Gran Área Metropolitana de Costa Rica. San José: Ministerio de Vivienda y Asentamientos Humanos.

Ministerio de Obras Públicas y Transporte. (2002). Tipos de transporte público colectivo según clasificación de servicio. San José: Ministerio de Obras Públicas y Transporte.

Molinero, A. \& Sánchez Arellano, L. (1997). Transporte público. México: Universidad Autónoma del Estado de México.

Programa de Investigación en Desarrollo Urbano Sostenible. (2014). Elaboración de auditorías de demanda y cálculo de indicadores y parámetros operativos del servicio de transporte remunerado de personas, modalidad autobús. San José: Universidad de Costa Rica.

Programa de Investigación en Desarrollo Urbano Sostenible. (2015). Elaboración de auditorías de demanda y cálculo de indicadores y parámetros operativos del servicio de transporte remunerado de personas, modalidad autobús que brindan el servicio en los corredores San José - Heredia y San José- Moravia. San José: Universidad de Costa Rica.

Programa de Investigación en Desarrollo Urbano Sostenible. (2016). Contratación de servicios profesionales para la elaboración de la I etapa del procedimiento para el cálculo de la demanda en las rutas de autobús por categorías, a partir de los siguientes componentes: (A) Las características de la ruta, (B) Las características del área que sirve y (C) Los indicadores de demanda. San José: Universidad de Costa Rica.

Pujol, Rosendo. (2002). Diferenciales entre zonas urbanas y rurales de Costa Rica: análisis estadístico de la información del Censo del 2000. San José: Programa de Investigación en Desarrollo Urbano Sostenible, Universidad de Costa Rica.

Salas, R. A. (2003). Regiones y cantones de Costa Rica. Serie cantones de Costa Rica, 2. San José: Instituto de Fomento y Ayuda Municipal.

Secretaría de Integración Económica Centroamericana (SIECA). (2011). Manual Centroamericano de Normas para el Diseño Geométrico de Carreteras. Ciudad de Guatemala: SIECA. 